\title{
Rauchverbote in Kneipen und Restaurants. Reaktion der Bürger und der gastronomischen Betriebe - Das Beispiel Düsseldorf
}

\section{Einleitung}

Seit dem 1. Juli 2008 gilt auch in Nordrhein-Westfalen ein Rauchverbot in Gaststätten. Damit ist eingetreten, was in den anderen Bundesländern schon früher - zum Teil bereits im August 2007 - realisiert wurde. Doch die öffentliche Debatte um Rauchverbote ist damit noch nicht abgeschlossen. Nach wie vor ist in der Öffentlichkeit kontrovers, wie im Einzelnen mit den Rauchverboten umzugehen ist und wie sehr zwischen den Bundesländern ein Harmonisierungsbedarf besteht. Was die Bevölkerung in der Landeshauptstadt von Nordrhein-Westfalen vom Rauchverbot hält, wie sich dies auf den Tabakkonsum auswirkt und wie sich die Umsetzung der Rauchverbote nach deren Inkrafttreten gestaltet, ist Gegenstand einer Fallstudie, die als Langzeituntersuchung angelegt ist und das Handeln von Menschen im Kontext sich ändernder Einstellungen und situativer Rahmenbedingungen untersucht. Die Frage, die insbesondere interessiert, ist, wie sehr äußere Restriktionen einen Einstellungswandel in der Bevölkerung begünstigen, informelle soziale Kontrollmechanismen eine zusätzliche Bedeutung erlangen und Raucher ihrem Bedürfnis nach Tabakgebrauch weiterhin folgen werden.

Wesentlicher Grundbestandteil der Untersuchung sind repräsentative Umfragen des Sozialwissenschaftlichen Instituts in der Düsseldorfer Bevölkerung. Sie beinhalten Fragen zu den Lebensverhältnissen in Düsseldorf und zu aktuellen Fragen, darunter auch zum Tabakgebrauch. Fragen zum Tabakgebrauch wurden von Beginn an (1997) in der Umfrageserie wiederholt gestellt, Fragen zu Rauchverboten waren erstmals 2004 in der Umfrage enthalten (einer Zeit, in der über Rauchverbote in Deutschland noch nicht in nennenswertem Maße diskutiert wurde). Die jüngste Erhebung in der Serie, durchgeführt Ende 2007 als Mehrthemenbefragung, basiert - ähnlich wie die früheren Erhebungen - auf einer Randomstichprobe aus dem Einwohnermelderegister. Sie stützt sich auf eine postalische Befragung mit bis zu drei Erinnerungsaktionen und erreichte nach Abzug neutraler Ausfälle (Zielperson verzogen, verstorben) eine Ausschöpfungsquote von 53 Prozent. Dies entspricht einer Quote, die für Befragungen - besonders in Großstädten - als überproportional hoch angesehen werden kann. 732 Befragte ab 18 Jahren mit deutscher Staatsangehörigkeit wurden befragt.

\section{Einstellungen der Bürger zu den Rauchverboten}

Im Gegensatz zu den meisten früheren Umfragen zum Thema wurden die Fragen zu den Rauchverboten in den Düsseldorfer Befragungen in differenzierter Form erhoben: So wur- 
de nicht global nach Rauchverboten in „Gaststätten“ gefragt, ${ }^{1}$ sondern die Einstellungen getrennt für „Restaurants“ und „Kneipen/Bars“ ermittelt. Des Weiteren wurde nicht global gefragt, ob man für „Rauchverbote“ sei, ${ }^{2}$ sondern für welche Art von Rauchverbot: für ein totales oder ein partielles mit Raucherzonen in Restaurants beziehungsweise Kneipen/ Bars. Eine derartige Differenzierung ist erforderlich, will man die Komplexität der Einstellungen zu diesem Thema und die Dynamik des zukünftigen Wandels in angemessener Form erfassen.

Tabelle: Einstellung der Düsseldorfer zum Rauchverbot in Restaurants und Kneipen (in Prozent)

\begin{tabular}{|l|c|c|}
\hline & Restaurants & Kneipen, Bars \\
\hline Vollständig verbieten & 43 & 20 \\
\hline Teilweise verbieten & 45 & 41 \\
\hline Nicht verbieten & 12 & 39 \\
\hline & 100 & 100 \\
\hline
\end{tabular}

Frageformulierung: „Man kann ja unterschiedlicher Ansicht darüber sein, an welchen Orten man das Rauchen verbieten sollte. Hier auf der Liste sind einige Orte aufgeschrieben. Was meinen Sie: Wo sollte das Rauchen vollständig verboten sein, teilweise verboten sein (durch Schaffung eigener Raucher-/ Nichtraucherbereiche) oder nicht verboten sein? ... Restaurants ... Kneipen/Bars".

Basis: repräsentative Umfrage in der Düsseldorfer Bevölkerung mit deutscher Staatsangehörigkeit; 18 Jahre und älter.

Angaben zur Erhebung: postalische Befragung, Erhebungszeitraum: Oktober und November 2007, Februar und März 2008.

Die Ergebnisse der Befragung (vgl. Tabelle) dokumentieren, dass sich eine Mehrheit der Bürger für Rauchverbote in Gaststätten ausspricht. Dieser Befund deckt sich mit den Befunden bundesweiter Umfragen, in denen global nach der Befürwortung von Rauchverboten gefragt wurde. Doch deutlich wird auch, dass es sich bei der Befürwortung keineswegs um eine generalisierte Befürwortung handelt und dass damit nicht zwingend totale Rauchverbote gemeint sind. Die Mehrheit nimmt vielmehr eine differenzierte Einstellung ein, sowohl was die Art der Gaststätte angeht als auch die Art des Rauchverbots: So plädierten 43 Prozent der Befragten für ein totales Rauchverbot in Restaurants, aber nur 20 Prozent für ein totales Rauchverbot in Kneipen/Bars. Entsprechend unterschiedlich groß ist auch der Anteil derer, die sich für kein Rauchverbot aussprechen: Mit Bezug auf Restaurants sind es lediglich zwölf Prozent, mit Bezug auf Kneipen/Bars mehr als dreimal so viel.

Dass zwischen Restaurants und Kneipen beziehungsweise Bars ein Unterschied gemacht wird, dürfte im Wesentlichen Folge der anders gearteten Funktionen sein, den diese Orte für deren Besucher einnehmen. Während man sich in Kneipen begibt, um dort gewöhnlich sein Bier zu trinken und mit anderen Menschen zu reden, geht es beim Restaurant um einen eher festlicheren Rahmen, bei dem das Essen im Vordergrund steht. Dass man anschließend (oder zwischendurch) zur Zigarette greift, kann vorkommen, ist aber gewöhnlich nicht so sehr Bestandteil des Lebensstils wie beim Kneipenbesuch. Wer sein Essen genießen möchte, will - selbst als Raucher - oft nicht durch den Rauch gestört werden, der von den Nachbarn herüberzieht.

\footnotetext{
1 Wie zum Beispiel beim Institut für Demoskopie (2008), Deutsches Krebsforschungszentrum (2008).

2 Wie zum Beispiel beim Institut für Demoskopie (2006), Deutsches Krebsforschungszentrum (2008a).
} 
Verständlicherweise befürworten Nichtraucher häufiger als Raucher ein Rauchverbot in Gaststätten. Doch bedeutet dies nicht zwangsläufig die Forderung nach einem totalen Rauchverbot: So sprechen sich lediglich 27 Prozent der Nichtraucher für ein derartiges Verbot in Kneipen/Bars aus, partielle Rauchverbote durch das Einräumen von Nichtraucherzonen finden bei ihnen mit 46 Prozent eher eine Befürwortung (weitere 27 Prozent bevorzugen keinerlei Verbot). Für Restaurants liegt der Anteil der Befürworter eines Totalverbots mit 55 Prozent zwar höher. Doch auch hier ist man derzeit von einer universalen Zustimmung zu einem vollständigen Rauchverbot entfernt. Eine derartige Situation ist geeignet, ambivalente Orientierungen in der Befolgung der neuen Gesetzgebung zu begünstigen.

Dass sich diese Verhältnisse in der Zukunft ändern und die Einstellungen der Bürger zu Rauchverboten verschärfen könnten, ist möglich (und wird von uns im Rahmen zukünftiger Replikationsuntersuchungen geklärt werden). Der Glaube, dass das Passivrauchen schädlich sei, ist jedenfalls inzwischen nahezu universal in der Bevölkerung verbreitet: In der Untersuchung stimmen unter den Nichtrauchern immerhin 62 Prozent voll und ganz dem Satz zu „Wer sich als Nichtraucher in einem Raum aufhält, wo Raucher sind, gefährdet seine Gesundheit“. Weitere 31 Prozent stimmen dem Satz „,eher zu“, lediglich sieben Prozent verneinen ihn (,eher nicht" beziehungsweise ,überhaupt nicht"). In dem Maße, wie Nichtraucher meinen, aktiv gegen das Passivrauchen etwas unternehmen zu müssen, könnte in der Bevölkerung in der Zukunft die Befürwortung strikter Rauchverbote steigen. Inwieweit die Rücksichtnahme auf Freunde und Bekannte, die sich weiterhin dem Tabakgebrauch hingeben, diesen Trend abmildert oder ihn gar aufhält, ist eine bislang ungeklärte Frage.

Die bisherigen Entwicklungen belegen jedenfalls eine abnehmende Tolerierung des Rauchens in den letzten Jahren. Dies gilt auch für das Rauchen in Gaststätten, und es betrifft hier Restaurants ebenso wie Kneipen und Bars: Meinten 2004 in Düsseldorf noch 41 Prozent der Nichtraucher, man solle in Kneipen/Bars das Rauchen generell erlauben, sind es jetzt nur noch 27 Prozent. Der Anteil derer, die ein totales Rauchverbot befürworten, ist parallel dazu von 14 auf 27 Prozent angewachsen. Selbst die Raucher sind diesem Trend in gewissem, wenn auch geringem Umfang gefolgt: Waren 200482 Prozent unter ihnen der Ansicht, man solle das Rauchen in Kneipen/Bars keinerlei Einschränkungen unterwerfen, sind es 2007 nur noch 65 Prozent. Die Akzeptanz partieller Rauchverbote ist damit allgemein - unter Nichtrauchern wie Rauchern - größer geworden. Dass sich diese steigende Akzeptanz nicht allein auf den gastronomischen Bereich bezieht, sondern allgemeinerer Art ist, dokumentieren die Ergebnisse zur Tolerierung des Rauchens an anderen Orten in unserer Untersuchung.

\section{Reaktionen der gastronomischen Betriebe auf die Gesetzgebung und die Herausbildung neuer Verhaltenszwänge für Raucher}

Welche Einflüsse aus den Einschränkungen der Rauchgelegenheiten auf den zukünftigen Tabakgebrauch erwachsen werden, ist ungewiss. Dass sich Nichtraucher, ermuntert durch die neue Gesetzgebung, häufiger als früher das Rauchen in ihrer unmittelbaren Umgebung verbitten, ist denkbar. Und denkbar (aufgrund ausländischer Erfahrungen auch wahrscheinlich) ist ebenfalls, dass Raucher aufgrund der neuen Zwänge vermehrt dazu moti- 
viert werden, den Konsum zu reduzieren oder gar einzustellen. Offen ist freilich auch, wie sehr es tatsächlich eine Kontrolle der Rauchgelegenheiten in Gaststätten geben wird - seitens der Ordnungsbehörden und der Nichtraucher, die intervenierend einwirken könnten.

Dass man seitens der Ordnungsbehörden zu wenig Personal hat und nicht aktiv auf eine gezielte Kontrolle ausgerichtet ist, ist in Düsseldorf wie anderswo offenkundig. ${ }^{3}$ Unter diesen Umständen hängt es maßgeblich davon ab, ob Nichtraucher von sich aus initiativ werden und Ordnungsbehörden auf Verstöße hinweisen. Die Tatsache, dass in den ersten zehn Tagen nach Erlass des Rauchverbots bereits rund 150 Beschwerden beim Ordnungsamt eingingen, ${ }^{4}$ zeigt, dass es durchaus heftige Reaktionen geben kann. Die Beschwerden, die vorgebracht wurden, betrafen Raucher in Kneipen und Gaststätten ebenso wie Raucherclubs.

Die Beschwerden kommen meist am Tag nach einem Gaststättenbesuch - und die Zigarette, um die es geht, ist zu diesem Zeitpunkt längst aus. Unter diesen Umständen ist es de facto unmöglich, Bußgelder gegen Raucher in Kneipen zu verhängen, und selbst gegen Wirte dürfte dies schwer fallen, beim Fehlen entsprechender Beweise. Bußgelder sind allenfalls möglich, wenn jemand ,,auf frischer Tat ertappt wird“. Und ob dies vorkommt oder nicht, ist letztlich von der Häufigkeit entsprechender Kontrollgänge des Ordnungsamtes abhängig. Bei rund 80 Kontrollen des Ordnungsamtes wurden in den zehn Tagen seit Inkrafttreten der Verordnung (lediglich) gegen drei Gäste, die geraucht hätten, Bußgelder verhängt worden. ${ }^{5}$ Wie sehr es in der Zukunft zu einer weiteren Zunahme der Anzeigen durch Nichtrauchergäste kommen wird (und ob sich womöglich gar ein Klima der „Denunziation“" ausbreitet) oder im Gegenteil eine recht schnelle Gewöhnung an Verstöße, ist eine offene Frage.

Obwohl das Gesetz zum Nichtraucherschutz schon seit langem angekündigt und in Nordrhein-Westfalen de facto am 1. Januar 2008 in Kraft trat - gastronomischen Einrichtungen wurde lediglich eine Schonfrist für etwaige bauliche Maßnahmen bis Ende Juni eingeräumt -, dürften viele Kneipen und Lokale relativ unvorbereitet in die neue Phase des Rauchverbots eingetreten sein. So antwortete auf eine Umfrage des Deutschen Hotelund Gaststättenverbandes Niederrhein vom März 2008 lediglich die Hälfte der angeschriebenen Betriebe, sie hätten bereits Maßnahmen zum Nichtraucherschutz getroffen. In den meisten Fällen handelte es sich um die Schaffung von Nichtraucherbereichen (71 Prozent) oder Raucherbereichen vor der Tür (23 Prozent). Rund 20 Prozent der Befragten, die Maßnahmen ergriffen hatten, hatten sogar bereits ein vollständiges Rauchverbot umgesetzt. ${ }^{6}$

Dass nicht mehr gastronomische Betriebe zu dieser Zeit bereits Maßnahmen in Gang gesetzt hatten, mag neben der Tatsache, dass einige als Einraumkneipen gar keine Möglichkeiten dazu hatten, auch mit der Vorstellung zu tun gehabt haben, dass man erst einmal die weitere Entwicklung abwarten müsse. Und rückblickend mag dies nicht einmal falsch gewesen sein: Denn gegenüber dem Bundesverfassungsgericht wurden zwischenzeitlich Beschwerden zum Nichtraucherschutz eingereicht. In ihnen klagten Besitzer von Einraumgaststätten gegen die Benachteiligung gegenüber Mehrraumgaststätten durch die bestehenden Gesetze. Eine Entscheidung, die für den 30. Juli 2008 anberaumt wurde, hätte

\footnotetext{
3 Vgl. Neue Rhein-Zeitung (01.07.2008), S. 1, und Westdeutsche Zeitung (01.07. 2008), S. 1.

4 Vgl. Rheinische Post (12.07.08), S. B1.

5 Vgl. Rheinische Post (12.07.2008), S. B1.

6 Vgl. Deutscher Hotel- und Gaststättenverband (2008).
} 
für die Raucher im ungünstigsten Fall die Folge haben können, dass jegliches Rauchen in Gaststätten verboten wird. Damit wären auch jegliche baulichen Maßnahmen durch Einrichtungen von Nichtraucherzonen vergeblich gewesen.

Dass die Besitzer von gastronomischen Betrieben dem Nichtraucherschutz eher skeptisch bis ablehnend gegenüberstehen, ist offenkundig. Denn nach einer Umfrage des Hotelund Gaststättenverbandes erwarten die meisten Umsatzeinbußen, ${ }^{7}$ und erste Ergebnisse aus anderen Bundesländern deuten in der Tat auch darauf hin (wie sehr dies auch längerfristig gilt, ist eine andere Frage). Die Reaktion vieler Einraumkneipen - aber auch Kneipen mit mehreren Räumen - auf die neue Gesetzgebung bestand in Düsseldorf und anderswo in der umgehenden Gründung eines „Raucherclubs“. Denn nach der Gesetzgebung für Nordrhein-Westfalen war nach den neuen Bestimmungen in Raucherclubs mit fester Mitgliedschaft das Rauchen erlaubt. Für die Düsseldorfer Altstadt wurde gar eine gemeinsame Clubmitgliedschaft angedacht, die eine Vielzahl von Kneipen zum Bestandteil eines Klubs umfasst.

Einer möglichen gesellschaftlichen Abwertung als verqualmter Ort zuvorkommend, stilisierten sich Raucherclubs recht bald in der Öffentlichkeit zum Ort kultureller Brauchtumspflege und Geselligkeit: mit einem Aufkleber, der vom Hotel- und Gaststättenverband vertrieben wird und der lautet: „Mensch. Kultur. Kneipe. Raucherclub.“ In der Tat fragt sich, wie sehr sich durch das gestiegene Bewusstsein um die Probleme des Passivrauchens in Kombination mit den neuen Restriktionen im öffentlichen Raum das Bild des Rauchers ändert: vom einstigen eher positiven Bild moderner Lebensführung hin zum negativ gefärbten Bild des Menschen, der unverantwortlich mit der eigenen Gesundheit und der Gesundheit anderer Menschen umgeht. Kneipen und Lokale, in denen sich bevorzugt Raucher einfinden, würden sich im Fall derartiger Bewertungsverschiebungen einem Imageverlust kaum entziehen können.

Dass die Etikettierung des Rauchens als soziale Abweichung in der Gesellschaft derzeit freilich noch nicht so weit fortgeschritten ist, dass der Besuch von Raucherkneipen bereits als etwas Ehrenrühriges gilt, dokumentiert die Berichterstattung über Rauchverbote in den Tageszeitungen. Hier erscheint die neue Gesetzgebung wie ein Eingriff, der die Freiheit des Einzelnen einengt. Und hier erschienen auch Ratschläge, wie man sich als Raucher den neu geschaffenen Restriktionen entziehen kann. So listeten die Düsseldorfer Tageszeitungen bereits am ersten Tag des Inkrafttretens der Verordnung auf, wo überall man in der Altstadt weiterhin rauchen könne. ${ }^{8}$ Und in der lokalen Ausgabe der Bild wurde gar ein offensiver Umgang für die Gastronomie propagiert: durch Bereitstellung eigener „BildAufkleber“ für gastronomische Betriebe mit der Aufschrift „Hier dürfen Sie rauchen Bild Kneipen Check“. 9

\footnotetext{
7 Vgl. Deutscher Hotel- und Gaststättenverband (2008).

8 So zum Beispiel die Rheinische Post (01.07.2008).

9 Vgl. Bild (01.07.2008), S. 4.
} 


\section{Aufnahmeformular \\ Raucherclub „Mensch. Kultur. Kneipe.“”}

Sehr geehrter Gast,

mit Ausfüllen und Unterschreiben dieses Aufnahmeformulars werden Sie Mitglied im

Raucherclub "Mensch. Kultur. Kneipe. -

in

$$
\text { (Ort) }
$$

“!

der seinen Stammsitz in dieser Gaststätte hat. Durch Ihre Mitgliedschaft können Sie an den regelmäßig stattfindenden Clubtreffen teilnehmen und den Vereinszweck, nämlich den gemeinschaftlichen Genuss von Tabakwaren, fördern.

Auszug aus der Satzung:

\section{§2 Zweck des Clubs}

Der Club verfolgt den ausschließlichen Zweck, den gemeinsamen Genuss von Tabakwaren in Nordrhein-Westfalen zu fördern. Dadurch soll die Rauchkultur sowie die gegenseitige Toleranz von Rauchern und Nichtrauchern sowie die

Rücksichtnahme von Rauchern auf Nichtraucher gefördert werden.

Die Mitgliedschaft ist kostenlos und zeitlich unbefristet; über die Einhaltung des satzungsgemäßen Zweckes hinaus entstehen für Sie keine weiteren Verpflichtungen. Sie können die Mitgliedschaft jederzeit kündigen.

Eine Kopie der Satzung ist kostenlos hier in der Gaststätte erhältlich.

Ja, ich werde Mitglied im Raucherclub „Mensch.Kultur.Kneipe. -

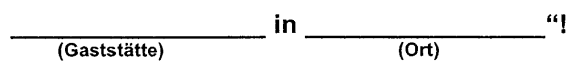

Name :

Geb.:

Straße:

PIz, Ort:

Ort, Beitrittsdatum

Unterschrift

Fassung vom 18.06.2008 


\section{Zukünftige Entwicklungstrends}

Mit der Entscheidung des Bundesverfassungsgerichts vom Juli 2008 darf in Gaststätten mit weniger als 75 Quadratmetern Fläche und ohne abgetrennten Nebenraum der Gastwirt das Rauchen gestatten - und zwar dann, wenn keine zubereiteten Speisen angeboten werden, der Eingangsbereich als Rauchergaststätte gekennzeichnet ist und Personen mit nicht vollendetem 18. Lebensjahr keinen Zutritt haben. Diese Regelung gilt bis zur Neuregelung, die der Gesetzgeber bis zum 31. Dezember 2009 zu treffen hat.

In welchem Umfang sich die Einraumgaststätten für die Lösung, Rauchergaststätte zu sein, entscheiden werden, bleibt abzuwarten - es ist auch eine Frage, ob ein Gastwirt dadurch seine bisherigen Gäste hält, verliert oder neue gewinnt, wie er selbst und das Personal dem Rauchen gegenüber eingestellt sind und ob Speisen zubereitet werden sollen. Vermutlich wird es sich um einen längerfristigen Prozess handeln, bei dem erst Erfahrungen gesammelt werden müssen. Dass die Zahl der Gaststätten, in denen geraucht werden kann, abnimmt, ist jedoch anzunehmen. Schließlich ist die Zahl der Nichtraucher in der Bundesrepublik größer als die der Raucher. Gleichwohl: Mag gesundheitspolitisch auch ein totales Rauchverbot die sinnvollste Lösung darstellen ${ }^{10}$ - Gelegenheiten zum Konsum von Zigaretten wird es weiterhin, wenn auch in eingeschränktem Maße, geben. Und die Kenntnis dieser Gelegenheiten wird weithin verbreitet sein. Die Debatte um das Für und Wider von Rauchverboten und deren Durchführung ist noch lange nicht beendet.

Dass Kampagnen Einstellungen ändern können und Verbote den Einstellungswandel bekräftigen, dafür gibt es unter anderem aus den USA Hinweise. So hatten zum Beispiel die Bürger Kaliforniens zu Beginn der 1990er Jahre ähnliche Einstellungen zu Rauchverboten wie die Einwohner in den anderen Landesteilen der USA. Mit der Initiierung von Anti-Raucher-Kampagnen kombiniert mit Rauchverboten in öffentlichen Räumen, einschließlich Restaurants und Bars, änderte sich dies jedoch: 1992-1993 sprachen sich in den USA (ohne Kalifornien) - ähnlich übrigens wie 2007 in Düsseldorf - lediglich 43 Prozent der Bürger für ein totales Rauchverbot in Restaurants aus, 1998-1999 war der Anteil geringfügig auf 48 Prozent gewachsen. Für ein totales Rauchverbot in Bars plädierten zu Beginn der 1990er Jahre 23 Prozent und am Ende der 1990er Jahre 27 Prozent. In Kalifornien hingegen, wo zwischenzeitlich eine intensive Anti-Raucher-Kampagne begonnen und Rauchverbote erlassen waren, lagen die Werte 1992-1993 für Restaurants bei 55 Prozent und 1998-1999 bei 71 Prozent, die analogen Werte für Bars lagen bei 27 Prozent 1992-1993 und 43 Prozent 1998-1999. ${ }^{11}$

Inwieweit sich in der Bundesrepublik in der Zukunft ein ähnliches Meinungsklima entwickeln wird, bleibt abzuwarten. Kennzeichnend für die Situation in Kalifornien (und manchen anderen Orten der USA) ist, dass sich im Gefolge der Anti-Raucher-Kampagnen vielfach eine Art „Remoralisierung“ des Tabakkonsums vollzogen hat: Ob jemand raucht oder nicht raucht, gilt nicht mehr als bloße Frage individueller Lebensführung und individueller rationaler Kosten-Nutzen-Kalkulation, sondern als eine Frage individueller und gesellschaftlicher Moral. ${ }^{12}$ Warum ein Verhalten moralisch „gut“" oder „schlecht“ ist, wird

\footnotetext{
${ }^{10}$ Vgl. Deutsches Krebsforschungszentrum (2006) und Deutsches Krebsforschungszentrum (2008b).

11 Vgl. Gilpin et al. (2004: 39).

${ }^{12}$ In manchen Orten der USA ist das Rauchen inzwischen in Parks und öffentlichen Plätzen verboten, selbst in Großstädten; in San Francisco zum Beispiel seit Januar 2005 (vgl. http://www.ntv.de (26.01.2005), in Los Angeles seit August 2007 (vgl. Los Angeles Times: „Mayor approves park smoking ban“,
} 
schließlich nicht mehr hinterfragt. Die „Entmoralisierung“ von Verhaltensweisen, wie sie sich in verschiedenen Verhaltensbereichen - auch in Bezug auf den Gebrauch illegaler psychoaktiver Substanzen - in den letzten Jahren vollzogen hat, ${ }^{13}$ stellt offenbar keinen zwangsläufigen, unumkehrbaren Trend moderner Gesellschaften dar. Unter welchen Bedingungen welche Maßstäbe mit welchen Begründungen an ein Verhalten angelegt werden und allgemeine Geltung in der Gesellschaft erlangen, ist bislang sozialwissenschaftlich freilich wenig erforscht.

\section{Literatur}

Deutscher Hotel- Und GASTSTÄTTENVERBAND (2008). Nichtraucherschutz. Erste Erfahrungen von Auswirkungen auf Gaststätten. Düsseldorf.

Deutsches KREBSFORSCHUngSZENTRUM (2006). Tabakbelastungen in deutschen Gastronomiebetrieben und in Fernreisezügen. Heidelberg.

DeUtsChes KREBSFORSCHUngSZENTRUM (2008a). Rauchfreie Gaststätten in Deutschland 2008: Mehr als zwei Drittel der Bevölkerung für eine bundesweit einheitliche Regelung, Aus der Wissenschaft - für die Politik. Heidelberg.

DEUTSCHES KREBSFORSCHUNGSZENTRUM (2008b). Gesundheitsschäden durch Rauchen und Passivrauchen. Fakten zum Rauchen. Heidelberg.

Gilpin, E. A., L. LEE und J. P. PIERCE (2004). „Changes in population attitudes about where smoking should not be allowed: California vs. the rest of the USA“, Tobacco Control 13, 38-44.

INSTITUT FÜR DEMOSKOPIE (2006). „Rauchverbote“. Allensbacher Berichte 12.

INSTITUT FÜR DEMOSKOPIE (2008). „Rauchverbote und Raucher“. Allensbacher Berichte 1.

REUBAND, K.-H. (1988). „Haschisch im Urteil der Bundesbürger. Moralische Bewertung, Gefahrenwahrnehmung und Sanktionsverlangen 1970-1987“, Neue Praxis. Zeitschrift für Sozialarbeit, Sozialpädagogik und Sozialpolitik 18, 480-495.

09.08.2007). Eine (unvollständige) Übersicht für Kalifornien findet sich unter http://ccap.etr.org/base/ documents/ListsofOTSPoliciesAdopted.doc. In manchen Orten gelten Rauchverbote auch für den Strand. So wurden entsprechende Bestimmungen 2003/2004 in den Orten Solana Beach, San Clemente und Santa Monica nahe Los Angeles erlassen. Inzwischen sind ebenfalls andere Städte betroffen (vgl. Rheinische Post Online 07.04.2004, Los Angeles Times: „Receding Tide for Beach Smokers“ 19.06.2006).

${ }^{13}$ Vgl. Reuband (1988). 